detection, such as midturbinate or saliva sampling, are on the horizon.

Acknowledgments. We are grateful to Michael Kulig, Elena Buckley, Andrew Risio, Jennifer Bryant, and Dr. Steven Brecher for their efforts in implementing our 3D-printing of nasopharyngeal swabs.

Financial support. No financial support was provided relevant to this article.

Conflicts of interest. All authors report no conflicts of interest relevant to this article.

\section{References}

1. The COVID tracking project website. https://covidtracking.com/. Accessed May 9, 2020.
2. Callahan CJ, Lee R, Zulauf KE, et al. Open development and clinical validation of multiple 3D-printed nasopharyngeal collection swabs: rapid resolution of a critical COVID-19 testing bottleneck. J Clin Micro May 2020 [Epub ahead of print]. doi: 10.1128/JCM.00876-20.

3. Nasal (anterior nasal) specimen collection for SARS-CoV-2 diagnostic testing. Centers for Disease Control and Prevention website. https:/www.cdc.gov/ coronavirus/2019-ncov/downloads/OASH-nasal-specimen-collection-factsheet.pdf. Accessed May 9, 2020.

4. Marty F, Chen K, Verrill KA. How to obtain a nasopharyngeal swab specimen. N Engl J Med 2020;382(22):e76. doi: 10.1056/NEJMvcm2010260.

5. Alcusky M, McManus DD, Hume AL, Fisher M, Tjia J, Lapane KL. Changes in anticoagulant utilization among United States nursing home residents with atrial fibrillation from 2011 to 2016. J Am Heart Assoc. 2019;8(9): e012023. doi: 10.1161/JAHA.119.012023.

\title{
Pediatric antimicrobial stewardship in the COVID-19 outbreak
}

\author{
Eneritz Velasco-Arnaiz MD ${ }^{1,2}$ (1), Maria Goretti López-Ramos PharmD, BCPPS ${ }^{1,3}$, Silvia Simó-Nebot MD ${ }^{1,2,4}$, \\ lolanda Jordan MD, $\mathrm{PhD}^{1,4,5,6,7}$, María Ríos-Barnés MD ${ }^{1,2}$, Mireia Urrea-Ayala MD, $\mathrm{PhD}^{1,8}$, Manuel Monsonís BSc ${ }^{1,9}$, \\ Clàudia Fortuny MD, $\mathrm{PhD}^{2,4,6,7}$ and Antoni Noguera-Julian MD, $\mathrm{PhD}^{2,4,6,7}$ on behalf of the Kids Corona project \\ ${ }^{1}$ Sant Joan de Déu Antimicrobial Stewardship Program (SJD-ASP), Sant Joan de Déu Hospital, Barcelona, Spain, ${ }^{2}$ Infectious Diseases Unit, Department of \\ Pediatrics, Sant Joan de Déu Hospital, Barcelona, Spain, ${ }^{3}$ Pharmacy Department, Sant Joan de Déu Hospital, Barcelona, Spain, ${ }^{4}$ Centre for Biomedical Network \\ Research on Epidemiology and Public Health (CIBERESP), Madrid, Spain, ${ }^{5}$ Pediatric Intensive Care Unit, Sant Joan de Déu Hospital, Barcelona, Spain, \\ ${ }^{6}$ Department of Pediatrics, University of Barcelona, Barcelona, Spain, ${ }^{7}$ Red de Investigación Translacional en Infectología Pediátrica, RITIP, Madrid, Spain, \\ ${ }^{8}$ Infection Control Department, Sant Joan de Déu Hospital, Barcelona, Spain and ${ }^{9}$ Clinical Microbiology Department, Sant Joan de Déu Hospital, \\ Barcelona, Spain
}

To the Editor-Growing evidence supports the positive impact of antimicrobial stewardship programs (ASPs) on antimicrobial use, including pediatrics. ${ }^{1}$ Although short of the level of acceptance these have reached in the United States, the implementation of pediatric ASPs in European hospitals has increased over the last few years. ${ }^{1}$

It has been suggested that the ASP should be helpful in the preparation for and response to the SARS-CoV-2/COVID-19 outbreak, ${ }^{2}$ but no formal recommendations have been published. Whether pediatric ASP remains an essential activity or not during the COVID-19 pandemic has yet to be clarified. Here, we describe how the COVID-19 pandemic has impacted antimicrobial use in a referral pediatric hospital, and we propose a supporting role for ASP teams in the local management of the outbreak.

The first COVID-19 case in Catalonia, Spain, was reported on February 25, 2020. By mid-March, most pediatric and obstetrics departments in the region were shut to increase the capacity for adult COVID-19 patients. Hospital Sant Joan de Déu Barcelona (SJD) remained the largest pediatric and maternal referral center in the region. COVID-19 and non-COVID-19 pediatric and young adult patients were transferred to our wards and pediatric ICU (PICU), and the number of daily deliveries tripled, whereas all nonemergency clinical, teaching, and research activities were

Author for correspondence: Eneritz Velasco Arnaiz, E-mail: evelasco@ sjdhospitalbarcelona.org

Cite this article: Velasco-Arnaiz E, et al. (2021). Pediatric antimicrobial stewardship in the COVID-19 outbreak. Infection Control \& Hospital Epidemiology, 42: 642-644, https:// doi.org/10.1017/ice.2020.312 postponed. Compared to the same months in 2019, in March 2020 , total hospital stays decreased by $0.8 \%$ in the PICU and $15.2 \%$ in non-PICU areas, and in April 2020, total hospital stays decreased by $23.7 \%$ in the PICU and $22.2 \%$ in non-PICU areas.

Following institutional recommendations, the SJD-ASP ${ }^{3}$ team reduced on-site work, but they continued to provide specific recommendations on individual antimicrobial prescriptions upon consultation by prescribers, and they monitored systemic antibiotic and antifungal use: days of-therapy (DOT) per 100 days present (DP). From March 16 to April 30 2020, 210 randomly selected prescriptions were assessed for quality. ${ }^{3}$

Because SARS-CoV-2 is a viral infection, it is not expected to directly influence antibiotic or antifungal use beyond the use of antibiotics with possible antiviral effect (ie, azithromycin) ${ }^{4}$ and the use of broad-spectrum antibiotics for superinfection in severe COVID-19 patients. ${ }^{5}$ However, we also observed antimicrobial use changes indirectly related to the outbreak. Antimicrobial use in March and April 2020 was significantly higher than in the same months in 2019 (Table 1). As expected, the use of azithromycin, included as first-line therapy in severe COVID-19 patients in combination with hydroxychloroquine, increased, particularly in the PICU. The use of ceftriaxone and teicoplanin, which were also prescribed at admission in severe COVID-19 cases, doubled in the PICU in April 2020 compared with April 2019. Other than ceftriaxone, antibiotics for community-acquired infections were prescribed less than in the same period in 2019, and cefazolin use decreased due to the dramatic drop in the number of surgeries. In contrast, the use of most broad-spectrum anti-gram-negative

(C) 2020 by The Society for Healthcare Epidemiology of America. All rights reserved. This is an Open Access article, distributed under the terms of the Creative Commons Attribution licence (http://creativecommons.org/licenses/by/4.0/), which permits unrestricted re-use, distribution, and reproduction in any medium, provided the original work is properly cited. 
Table 1. Total Antimicrobial Use (AU) and Selected Antibiotic (Ab) and Antifungal (Af) Use in February, March, and April 2019 and 2020, in Days of Therapy (DOT) per 100 Days Present (DP) in PICU and Non-PICU Areas (Excluding Operating Rooms) ${ }^{\mathrm{a}}$

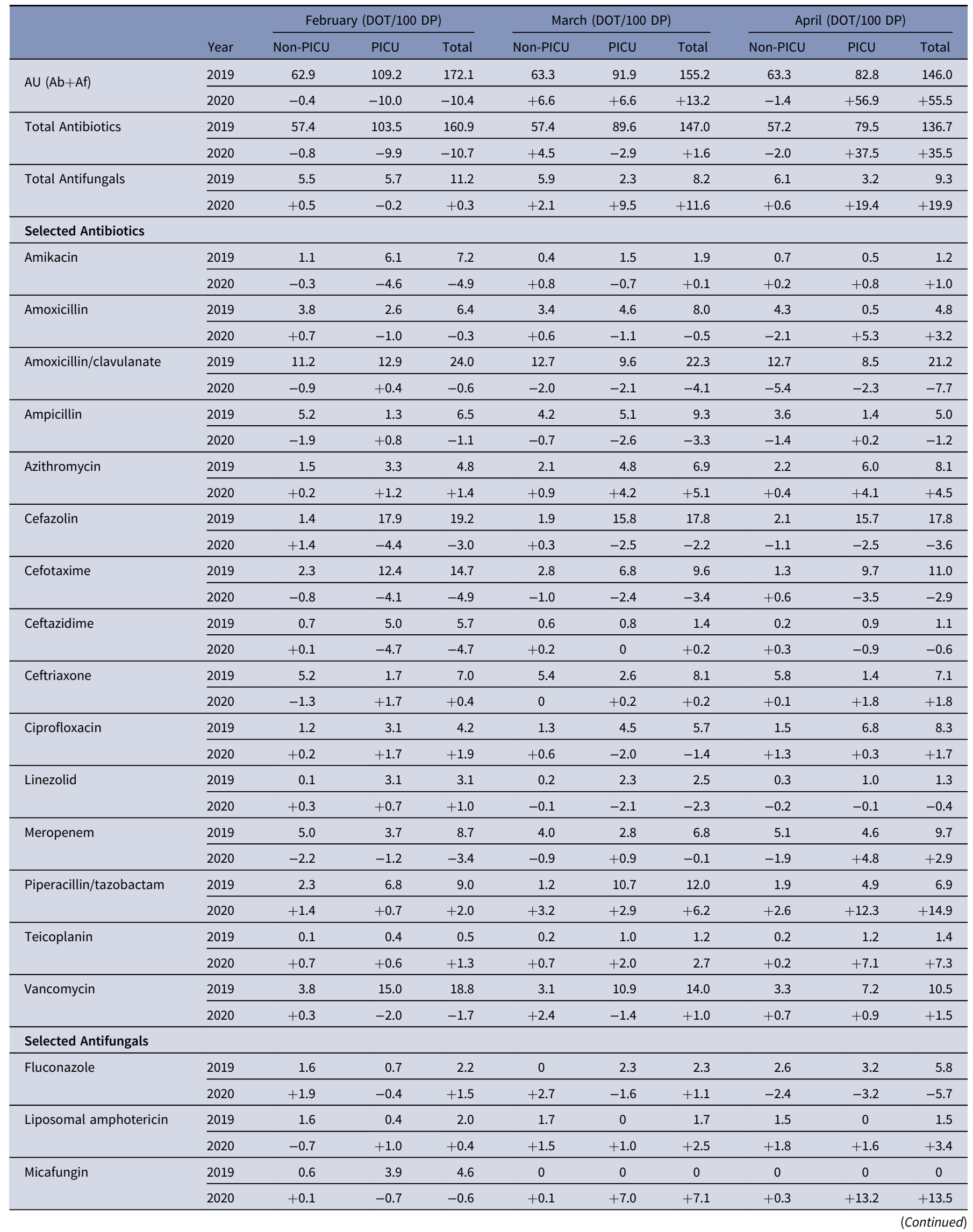


Table 1. (Continued)

\begin{tabular}{|c|c|c|c|c|c|c|c|c|c|c|}
\hline & \multirow[b]{2}{*}{ Year } & \multicolumn{3}{|c|}{ February (DOT/100 DP) } & \multicolumn{3}{|c|}{ March (DOT/100 DP) } & \multicolumn{3}{|c|}{ April (DOT/100 DP) } \\
\hline & & Non-PICU & PICU & Total & Non-PICU & PICU & Total & Non-PICU & PICU & Total \\
\hline \multirow[t]{2}{*}{ Posaconazole } & 2019 & 1.0 & 0 & 1.0 & 0.3 & 0 & 0.3 & 1.2 & 0 & 1.2 \\
\hline & 2020 & -0.5 & +0.4 & 0 & +1.2 & +0.3 & +1.5 & +1.0 & +0.7 & +1.6 \\
\hline \multirow[t]{2}{*}{ Voriconazole } & 2019 & 0.7 & 0.7 & 1.4 & 1.4 & 0 & 1.4 & 0.4 & 0 & 0.4 \\
\hline & 2020 & -0.3 & -0.7 & -1.0 & -1.0 & +2.8 & +1.8 & +0.2 & +7.2 & +7.3 \\
\hline
\end{tabular}

Note. Ab, antibiotic; Af, antifungal; AU, antimicrobial use; DOT, days of therapy; DP, days present; PICU, pediatric intensive care unit.

a Year 2020 data are given as increases or decreases compared to the same month in year 2019.

drugs with anti-Pseudomonas activity rose in the PICU, and piperacillin-tazobactam and ciprofloxacin use also increased in non-PICU patients. These changes were probably related to the transfer of patients with immunosuppressive and/or other complex conditions from other centers that had shut their pediatric departments. Similarly, the rise in micafungin use and, to a lesser extent, voriconazole use, was likely related to the transfer of oncological and hematopoietic stem cell transplantation (HSCT) patients already on antifungal prophylaxis or antifungal treatment in accordance with other institutions' local protocols. The temporary modification of our antifungal prophylaxis protocol for oncology patients (inhaled liposomal amphotericin plus oral fluconazole) meant minimizing the use of nebulized drugs and led to an increase in intravenous liposomal amphotericin use.

Despite major changes in antimicrobial use, we have not observed a critical deterioration of antimicrobial prescription quality to date. Of the 210 evaluated prescriptions, 167 (79.5\%) were considered 'optimal' in accordance with current protocols, compared with 316 of $400(79.0 \%)$ in the same period in 2019. However, continuous monitoring allowed the identification of high workload areas deserving enhanced support, like involving the care of oncology patients.

In the context of a pandemic, changing clinical circumstances may negatively affect the quality of antimicrobial prescriptions; prescribers have to work outside their comfort zone, dealing with a new disease ${ }^{6}$ and/or 'unusual' patient profiles (ie, young adults or pregnant women in a PICU in our case). New COVID-19 protocols are constantly updated in accordance with newly available information, and previous protocols have been temporarily modified in favor of medical nonsurgical management of some conditions (eg, noncomplicated appendicitis).?

Our data show that the SARS-CoV-2 pandemic has the potential to have a significant impact on antimicrobial use in the pediatric inpatient population; pediatric ASP monitoring and interventions remain useful to preserve the quality of prescriptions, at least in the short term. However, the COVID-19 pandemic is still ongoing, as are other non-COVID-19 health issues such as AMR, ${ }^{5}$ so healthcare resource distribution and organization in the post-COVID-19 period are uncertain. Planning the response to epidemic waves should include the widespread integration of ASP, with (1) involvement of the ASP team in guidelines development as exemplified in "Multicenter Initial Guidance on Use of Antivirals for Children with COVID-19/SARS-CoV-2"; ; (2) integrated response of common ASP local and external partnerships and infrastructures, including structured interviews, formularies, and other information technology tools' ${ }^{9}$ and (3) coordination of indicator selection and monitoring routines to support a continuous evaluation strategy.

If pediatric ASPs have suffered some weakening in the current crisis, they should be reinforced promptly to sustain high-quality care, maintaining the principles of antibiotic stewardship. ${ }^{5}$ The potential benefit of a more active role for pediatric ASPs in the outbreak response, above and beyond their regular activities, should be taken into consideration.

\section{Acknowledgments.}

Financial support. This research did not receive any specific grant from funding agencies in the public, commercial, or not-for-profit sectors. Data were generated as part of the routine work of Hospital Sant Joan de Déu.

This work was supported by "Contratos Río Hortega. Convocatoria 2018" (Acción Estratégica de Salud. Ayudas y Subvenciones. Instituto de Salud Carlos III. Ministerio de Ciencia e Innovación. Spain, grant no. CM18/00054 to S.S.N.) and by "Subvencions per a la Intensificació de Facultatius Especialistes" (Departament de Salut de la Generalitat de Catalunya, Programa PERIS 2016-2020, grant no. SLT008/ 18/00193 to A.N.-J).

Conflicts of interest. S.S.N. and C.F. have received funds for speaking at symposia organized on behalf of Gilead Sciences. All other authors have no conflicts of interest to disclose.

\section{References}

1. Donà $\mathrm{D}$, Barbieri $\mathrm{E}$, Daverio $\mathrm{M}$, et al. Implementation and impact of pediatric antimicrobial stewardship programs: a systematic scoping review. Antimicrob Resist Infect Control 2020;9:3.

2. Stevens MP, Patel PK, Nori P. Involving antimicrobial stewardship programs in COVID-19 response efforts: all hands on deck. Infect Control Hosp Epidemiol 2020;41:744-745.

3. Velasco-Arnaiz E, Simó-Nebot S, Ríos-Barnés M, et al. Benefits of a pediatric antimicrobial stewardship program in antimicrobial use and quality of prescriptions in a referral children's hospital. J Pediatr 2020 Jun 6 [Epub ahead of print]. S0022-3476(20)30709-5. doi: 10.1016/j.jpeds.2020. 06.008 .

4. Damle B, Vourvahis M, Wang E, Leaney J, Corrigan B. Clinical pharmacology perspectives on the antiviral activity of azithromycin and use in COVID-19. Clin Pharmacol Ther 2020 Apr 17 [Epub ahead of print]. doi: 10.1002/cpt.1857.

5. Huttner B, Catho G, Pano-Pardo JR, Pulcini C, Schouten J. COVID-19: don't neglect antimicrobial stewardship principles! Clin Microbiol Infect 2020; 26:808-810.

6. Lai J, Ma S, Wang Y, et al. Factors associated with mental health outcomes among health care workers exposed to Coronavirus disease 2019. JAMA Netw Open 2020 Mar 2 [Epub ahead of print]. 3:e203976. doi: 10.1001/jamanet workopen.2020.3976.

7. Huang L, Yin Y, Yang L, Wang C, Li Y, Zhou Z. Comparison of antibiotic therapy and appendectomy for acute uncomplicated appendicitis in children: a meta-analysis. JAMA Pediatr 2017;171:426-434.

8. Chiotos K, Hayes M, Kimberlin DW, et al. Multicenter initial guidance on use of antivirals for children with COVID-19/SARS-CoV-2. J Pediatric Infect Dis Soc 2020 Apr 22 [Epub ahead of print]. pii: piaa045. doi: 10.1093/ jpids/piaa045.

9. Stevens RW, Estes L, Rivera C. Practical implementation of COVID-19 patient flags into an antimicrobial stewardship program's prospective review. Infect Control Hosp Epidemiol. 2020 Apr 15 [Epub ahead of print]. doi: 10 1017/ice.2020.133. 